\title{
Increased Percentage of $\beta$-Thalassemia Carriers in the Region of South-East Rodopi and Kardzali Near Greece and the Mediterranean
}

\author{
Violeta Yordanova ${ }^{1}$, Todor Tcherkezov ${ }^{2}$ \\ ${ }^{1}$ Molecular Diagnostics Unit, MPHAT “Dr. Atnas Dafovski”, Kardzali, Bulgaria \\ ${ }^{2}$ Department of Public Health, Medical University, Sofia, Bulgaria
}

Email address:

yordanovavioleta@yahoo.com (V. Yordanova)

\section{To cite this article:}

Violeta Yordanova, Todor Tcherkezov. Increased Percentage of $\beta$-Thalassemia Carriers in the Region of South-East Rodopi and Kardzali Near Greece and the Mediterranean. International Journal of Cardiovascular and Thoracic Surgery. Vol. 7, No. 3, 2021, pp. 41-45. doi: $10.11648 /$ j.ijcts.20210703.12

Received: June 16, 2021; Accepted: July 2, 2021; Published: September 30, 2021

\begin{abstract}
Objectives: In a brief summary we present the basic aspects of the of the hemoglobinopathies in the population of the South-East Rodopi within the Bulgarian territory and we report the preliminary results for the identification of $\beta$ thalassemia carriers by measuring the expression of the HbA fractions. Design and methods: Separation and measurement of the $\mathrm{Hb}$ fractions in blood samples collected among the multi-ethnic Bulgarian population using different methods. Results: By using a cut of $3.2 \% \mathrm{HbA}_{2}$ we have found $9.77 \%$ carriers of point mutations defects among a group of 532 patients of different ethnic origin. All patients' carriers have also increased HbF. Most frequent point mutation is IVS I nt 110 (G>A). Discussion: All methods summarized in this paper provide identification at practically high sensitivity and specificity. However, all methods should be followed by routine parent's analysis to confirm the provisional results. Taking into consideration the specific conditions of each patient and the $\mathrm{HbA}$ expression, we believe carriers of $\beta$-thalassemia can be preselected with a reasonable degree of sensitivity and be confirmed by parent analysis. It is important to consider this uneven distribution of the mutations in the hemoglobin being increased in our region as opposed to the overall carriers in the territory of Bulgaria and Europe.
\end{abstract}

Keywords: Mediterranean Anemia, $\beta$-Thalassemia, $\mathrm{HbA}_{2}$, Hemoglobin Mutation Carriers

\section{Introduction}

Among the hemoglobinopathies the thalassemia is a specific type in which the circulating hemoglobin $\mathrm{HbA}$ fractions are over $96 \%$ of the hemoglobin in adults and consist of 2 alfa and 2 beta chains ( $\alpha 2 \beta 2)$; tetrameres. $\beta$ Thalassemias are of a particular interest to us due to the higher frequency in our region. The other hemoglobionopathies are either very rare or not found here at all [1]. They are coded from $4 \alpha$ and $2 \beta$ genes. Known and described as of current 600 variant permutation of autozomal-recessive defects to these genes lead to broken structure of $\beta-\mathrm{Hb}$ (Hemoglobinoses) or to defects in the synthesis of $\beta$ or $\alpha$-chains of the globin molecule and are $\beta$ or $\alpha$-thalassemias, respectively.

Most recently the new approaches and scientific understanding of the regulatory mechanisms of the hemopoesis gives hope for the patients with heavy mutations. To this date there are still 10000 registered homozygous careers in Europe and USA [2]. It has been found the condition is in link to the malaria fever and the spread of the mosquito population, therefore we see it predominantly in the Mediterranean, North Africa, Middle East, Arabic Peninsula, India and Indonesia. In some regions the frequency can reach from $0,1 \%$ in Eastern Europe to $16 \%$ in Cyprus, as the frequency of the affected fetuses on 1000 pregnancies varies between 0,0003 to 5,12 in Cyprus [3]. 


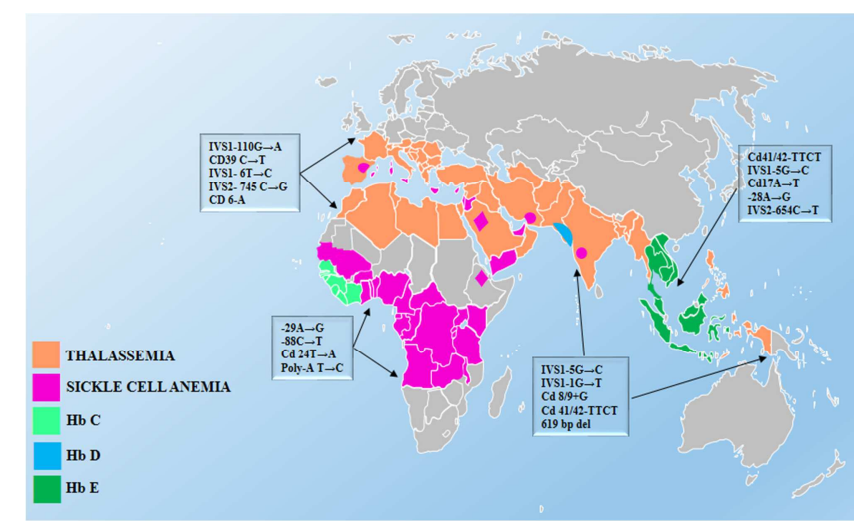

Figure 1. Distribution of the thalassemia and most common mutations of $\beta$ thalassemia in different countries [4].

In clinically asymptomatic patients there is no need of specific therapy. Many different mutations may cause $\beta$ thalassemia, inherited as many genetic combinations, determining heterogenic group of clinical syndromes, which may or may not present. In thalassemia minor, very common as $\beta$-thalassemia or heterozygous $\beta$-thalassemia, there is one single $\beta$-thalassemic mutation and a normal $\beta$-globin gene on the other chromosome. Microcytosis, hypochromia, high level of $\mathrm{HbA}_{2}$ and weak or minimal anemia are the characteristics of this type of thalassemic carriers, increased number of erythrocytes, lower MCV and $\mathrm{MCH}$. On a blood smears is evident microcytosis and hypochromia, anysocytosis, poykilocytosis and nuclear red blood cells (erythroblasts) [5].

The anemia of Thalassemia Major is so severe, that there is a need of constant blood transfusion, increasing the absorption of iron lead to deposition of iron ions in many organs (liver, endocrine tissue, heart) and the endpoint could be lethal, if not helatotherapy used [5]. Analysis of hemoglobin fractions show mainly $\mathrm{HbF}$ plus some amount $\mathrm{HbA}_{2}$ in the $\beta^{\circ}$ homozygous, but not enough to compensate for the loss of the $\mathrm{HbA}$, so the $\mathrm{Hb}$ level remain $<5 \mathrm{~g} / \mathrm{dL}$ [5]. Most $\beta^{+}$homozygous and $\beta^{+} \beta^{\circ}$ combined heterozygous also have thalassemia major. $\mathrm{HbF}$ remains dominant component, but the varied amount $\mathrm{HbA}$ depends on the specific $\beta^{+}$ thalassemic allele [5]. $\beta$-Thalassemia intermedia (TI) is referred to the less severe phenotype, in which there is a significant anemia, but chronic therapy of blood transfusion is not always required. The patients show definitive heterozygous picture, varyingly in severity from the $\beta$ thalassemic carriers to the point of thalassemia major [6]. Most patients with the thalassemia intermedia are homozygous or a combination of heterozygous for $\beta$ thalassemia, as with both $\beta$-globin loci affected. Less frequently are the ones with only one locus of $\beta$-globine affected and the other normal. The mild clinical characteristics of TI in comparison to thalassemia major are due to the inheritance of two $\beta$-thalassemic mutations, one light and one heavy; the inheritance of two light mutations or sometimes the caring over more complex combinations, as for instance two $\beta$-thalassemic mutations co-inherited with heterozygous $\alpha$-thalassemia. In the later form, also common as $\alpha \beta$-thalassemia, $\alpha$-thalassemic allele lowers the heavy impact of unbond $\alpha$-globin chains [7-10].

$\beta$-Globin gene is at the short arm of the chromosome 11 and it is roughly about $80 \mathrm{~Kb}$ long, which contains also $\delta$ globin gene, the embryonic $\varepsilon$ gene, the fetal $A \gamma$ gene and $\mathrm{G} \gamma$ gene $[1,8]$. The five functional globin genes are arranged in the chromosome in the order of their expression $[1,8]$.

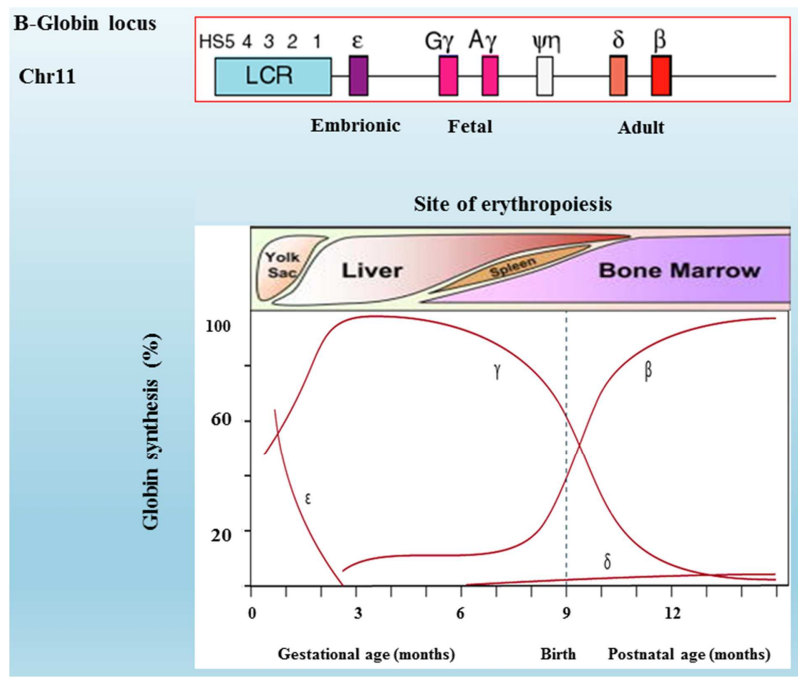

Figure 2. The above scheme presents the construction of the human $\beta$-globin locus. The lower part of the diagram shows the transition from fetal to adult form of hemoglobin. The normal time lapse is being presented [4].

Clinically $\beta$-thalassemia come from mutations, which involve each and every step of the formation of the globin molecules; the transcription, processing of mRNA, translation of mature mRNA and post-translational modifications of the $\beta$-polypeptide chain [12].

Around 200 different alleles of the $\beta$-globin gene are being found and described, the vast of them are the point mutations or immediate flanking sequence. The distribution of these alleles varies significantly in the different populations, but within a single population there are only few alleles (Figure 1) [4], most alleles are Mediterranean recessive. Conditions with a greater syntheses of $\mathrm{HbF}$ for a compensation are common as inherited stable conditions of fetal hemoglobin $(\mathrm{HPFH})$ and the homozygous are clinically unaffected, while the heterozygous with the $\beta$-thalassemia are very mild [5]. In normal individuals only $5-8 \%$ of the erythrocytes, called the $\mathrm{F}$ cells contain $\mathrm{HbF}, 5-20 \%$ from the total hemoglobin in those particular cells $[10,11]$.

The treatment with stem cells is the available option for patients with Thalassemia Major and potential risks of death and complications. Transplantation of blood from an umbilical cord have a limited success, because large volumes are required for the upkeep of the hemopoesis. Genetic therapy is in a phase of early clinical trials and offers excellent perspectives. Induction of synthesis of $\gamma$-globin chains through genetic transduction or pharmacologically has a potential benefit for patients with $\beta$-thalassemia $[12,13$, and 14]. Newer methods for treatment of TI and TM were being published with the use of $\mathrm{Tf}$, hepsidin agonists and 
JAK2 inhibitors [6].

There is still quite a stigma around this particular condition; many people lead their life having no idea. The experiential knowledge of affected individuals and families is a significant, yet under-utilised, resource that may be harnessed in the context of genetic counselling in order to address some of these difficulties. It has been suggested that insights from affected families could be imparted to genetic counselling patients through a variety of means; for example, through personal stories, photographs, vignettes, videos and interviews, all of which may assist in humanising and "bringing to life" a genetic condition. Support and advocacy groups for already affected families may be a particularly important resource in developing and evaluating these resources. There is limited published data exploring the usage of these groups by individuals and couples identified as carriers, particularly those facing complex decisions about invasive diagnostic testing and/or selective pregnancy termination. The lack of correspondingly well - developed mechanisms of information and support within the groups for genetic disorders, such as thalassaemia, may be explained by the relative rarity of these conditions individually, the (already strained) resources of such groups and also by the somewhat ambiguous character of carrier status. Carrier status have been considered analogous to a liminal state, a halfway house between health and disease, the significance of which can be difficult to interpret. Carrier couples may struggle to reconcile this ambiguity in their identities which, in turn, may render them alienated from such groups, the ethos of which may also clash with their own reactions to their genetic status [16].

Some scientific groups prefer initial screening method [17] by phone interviews, others utilize more in-depth approach and soon enough the implementations of CRISPR technology might be a possible choice for patients with identified mutations in the hemoglobin gene [18].

In Bulgaria the percentage of thalassemia is roughly 2.5$3 \%$. Annually we have $12-15$ children born with a form of a heavy major clinical appearance $[1,14]$.

We previously found increased percentage of carriers for thalassaemia mutations in the region of Kardjali and near the border crossings [15]. We suspected to find higher percentage of point mutations in our region to be more likely similar to those in Turkey and Greece, due to the near proximity of Mediterranean, the intermixture of the population, the ongoing migration, etc.

\section{Materials and Methods}

We collected blood in EDTA (K3E) vacutainers, routine $\mathrm{CBC}$ done per protocols and further $40 \mu \mathrm{l}$ sample used to for either gel electrophoresis or HPLC method. The samples were either native or precipitated hemolysed erythrocytes mixed with loading gel buffer and loaded on SDS PAGE gel. The gels were run and further stained with Coomassy blue, distained with methanol/acetic acid buffer (BioRad). Each gel was done with controls (positive and negative) and protein marker - Precision Plus Protein Standards - Dual Color (BioRad). The gels were further analyzed by Photo Capt Doc Print II gel imaging system for percentage of hemoglobin fraction estimation.

The high performance liquid chromatography was done via HPLC stationary system for clinical diagnostics (BioRad); $\mathrm{D}-10$ utilizing the principle of ion-exchange high performance chromatography as a referent method for hemoglobin fractions percentage. Genome DNA was isolated for mutation study. We tested the newly developed method from SNP Biotechnology Ltd. Ankara, Turkey for determination of possible six most common point mutations in the hemoglobin gene: IVS1-1, IVS1-110, IVS2-1, IVS2745, IVS1-6 and Sickle cell. For the genomic DNA isolation we used QIAamp DNA blood mini kit from Qiagen. For the PCR reaction we used AB StepOne rtPCR system.

\section{Patients}

We subjected to a test for $\beta$-thalassaemia carriers 532 patients from our hospital. They were randomly selected and came from different departments, they were not discriminated whether outpatients or in hospital stay. The patients had had either electrophoresis or HPLC blood test in addition to the routine $\mathrm{CBC}$ at the hospital, subsequent to their informed consent. The patients were between the age of 5 and 87 , male and female (39-61\% male to female ratio)

\section{Results}

The obtained results we present in figure 3, 4 and 5. Our current extensive data shows higher percentage of carriers$9.774 \%$. The results from the rest of the parameters - the $\mathrm{CBC}$, are as follows:

Table 1. Mean results of the blood parameters in patients carriers for thalassaemia.

\begin{tabular}{clllllll}
\hline age & Hb & Ht & MCV & MCH & MCHC & HbA $_{2}$ & HbF \\
\hline Mean data 46.79 & 111.29 & 0.370 & 73.88 & 22.33 & 300.76 & 3.68 & 1.83 \\
\hline
\end{tabular}

\section{Carriers b-Thalassemia}

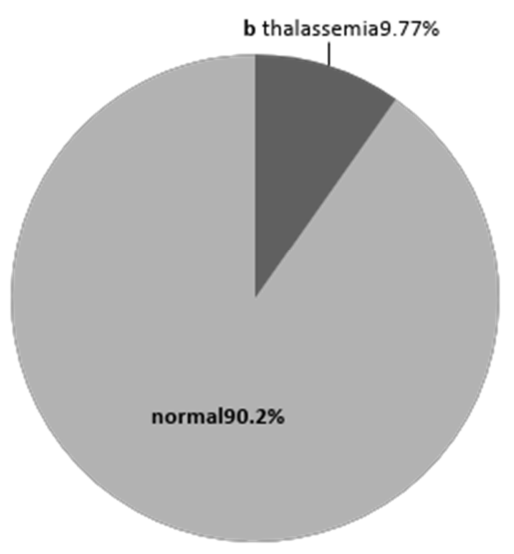

Figure 3. Distribution of thalassemia careers (\%). 
The carriers also show increased percentage of $\mathrm{HbF}$ $(1.83 \%)$, varying between 9.8 and $0.8 \%$.

Out of 532 patients, 48 had results $\mathrm{HbA}_{2}$ of $3.2 \%$ (9\% of the total number) - result at the upper limit range of the reading, considered normal.

Two patients had a typical percentage of hemoglobin fractions as bands on the gel for Delta-Beta Thalassemia intermedia, presented as $0.3 \%$ of the total.

According to their ethnic background, we had: - $61.7 \%$ Turkish, $38.1 \%$ Bulgarian and $0.2 \%$ other. In carriers the ethic distribution was: $56 \%$ Turkish, $42 \%$ Bulgarian and $2 \%$ other (figure 4).

\section{Ethnicity carrier patients}

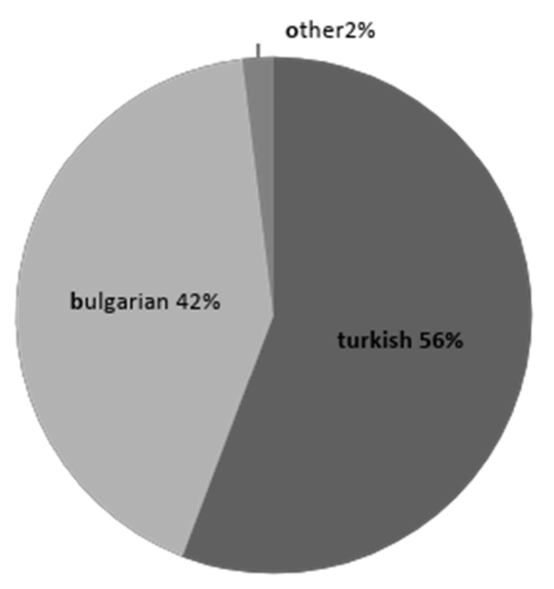

Figure 4. Distribution of thalassemia careers with regards of the ethnic origin (\%).

Interesting finding presented the types of specific mutations and their distribution. To this point we have the greatest percentage of IVS1-110 (45\%), IVS2-745 (42\%), and IVS1-6 (10\%), other (3\%). Our data trend shown from the mutations prevalence was in accordance to the previously reported distribution in Bulgaria, [1].

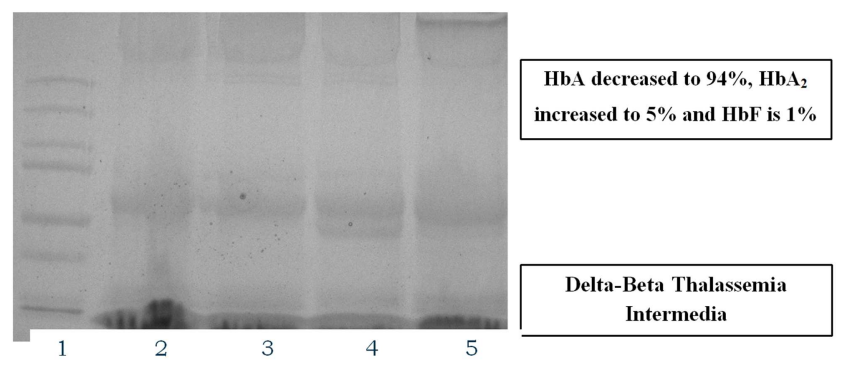

Figure 5. Image of gel, stained with Comassie Blue, showing the different patterns in bands with carriers of thalassaemia mutations. Line 1 is loaded with Precision Plus Protein Standards, 2-negative control, 3-b-thalassemia carrier, 4-Delta-Beta Thalassemia, 5-normal patient.

\section{Discussion}

The presented results above show increased percentage carriers in our region and our study is important evidence toward the local gene distributions. We provide evidence our region has greater genetic similarities in the hemoglobin gene with the populations near Mediterranean, than the rest of Bulgaria. Further research is necessary to collect more data for the carriers, establish registry for patients and their families. Such registry is of essential importance to the patients, providers and specialists. Our initial research might give foundation for improving hospital methods and implementing them. The potential great benefit to the public health is to establishing programs with methods for identifying carriers with over $2.5 \%$ per given population, and utilizing prophylaxis methods of Cyprus, Greece, Italy, UK as per WHO [2-4]. A routine rtPCR testing for our patients would be preventive in family planning; moreover will give us information in revealing the mutations and opportunity for laying out options to our patients. For patients with $\mathrm{HbA}_{2}$ on the upper range of $3.2 \%$ more in depth investigation with the rtPCR methods to determine whether specific mutation in the hemoglobin is in the etiology could reveal their overall condition, family history and underlying problems.

We think of our research as an important element in all efforts to further cut the costs for constant therapies of permanent conditions; therefore invest in preventive classes for parents, informational brochures, materials and seminars to educate the local population. Our study might be an important step forward in terms of connecting the patients with anemia and hemoglobinopathies with the contemporary methods for diagnostics, therapy and social support.

\section{Conclusion}

Our research might be important in revealing the prevalence of carriers near the Mediterranean, also sharing common mutations with the population of Greece and Turkey. We give our modest contribution toward the thalassaemia distributions and the migrations, which might be important for improvement of life quality of the affected patients and their overall social status in underdeveloped regions of the South-East Rodopi.

\section{References}

[1] Petkov, Georgi \& Yordanov, Georgi \& Chakarov, Ivan \& Vassileva, Petya \& D. Efremov, Georgi. (2013). Molecular Basis of -thalassaemia in Bulgaria. An Update. Proceeding of the Bulgarian Academy of Sciences. 66. 10.7546/CR-2013-664-13101331-17.

[2] Modell, Bernadette; Darlison, Matthew - Global epidemiology of haemoglobin disorders and derived service indicators Bulletin of the World Health Organization http://www.who.int/bulletin/volumes/86/6/06-036673/en/.

[3] Bozkurt G22 Results from the north cyprus thalassemia prevention program. Hemoglobin. 2007; 31 (2): 257-64.

[4] Nadia Maria Sposi Medicine «Hematology» Chapter 4 Interaction between Erythropoiesis and Iron Metabolism in Human $\beta$-thalassemia - Recent Advances and New Therapeutic Approaches 2015. 
[5] Palstra RJ, de Laat W, Grosveld F. $\beta$-globin regulation and long-rage interactions. Adv Genet. 2008; 61: 107-142.

[6] Weatherall DJ, Clegg JB, Higgs DR and Wood WG. The hemoglobinopathies. In: Scriver CR, Beaudet AL, Sly WS, Valle D, Vogelstein B, editors. The Metabolic and Molecular Bases of Inherited Disease (OMMBID); Chapter 101. New York, NY: McGraw-Hill. 2002.

[7] Galanello R, Cao A. Relationship between genotype and phenotype. Thalassemia intermedia. Ann NY Acad Sci. 1998; 850: $325-333$.

[8] Weatherall D. The molecular basis for phenotypic variability of the common thalassemias. Mol Med Today. 1995; 1: 15-20.

[9] Camaschella C, Mazza U, Roetto A, Gottardi E, Parziale A, Travi M, Fattore S, Bacchiega D, Fiorelli G, Cappellini MD. Genetic interactions in thalassemia intermedia: Analysis of beta-mutations, alpha-genotype, gamma-promoters, and betaLCR hypersensitive sites 2 and 4 in Italian patients. Am J Hematol. 1995; 48 (2): 82-87.

[10] Cappellini MD, Musallam KM, Cesaretti C, Taher A. Thalassemia intermedia. ESH Handbook on Disorders of Herythropoiesis, Erythrocytes and Iron Metabolism. 2009; chapter 12: 286-309.

[11] Li Q, Peterson KR, Fang X, Stamatoyannopoulos G. Locus control regions. Blood. 2002; 100: 3077-3086.

[12] Nienhuis AW, Nathan DG. Pathophysiology and clinical manifestations of the $\beta$-thalassemias. Cold Spring Harb Perspect Med. 2012 Dec. 1; 2 (12): a011726.

[13] Boyer SH, Belding TK, Margolet L, Noyes AN. Fetal hemoglobin restriction to a few erythrocytes ( $F$ cells) in normal human adults. Science. 1975; 188: 361-363.

[14] Petkov, Georgi \& D. Efremov, G \& Efremov, Dimitar \& Aleksandar, Dimovski \& Tchaicarova, P \& Tchaicarov, R \& Rogina, B \& Agarwal, Sarita \& Kutlar, Abdullah \& Kutlar, Feride \& L. Reese, A \& A. Stoning, T \& H. J. Huisman, T. (2009). $\beta$-Thalassemia in Bulgaria. Hemoglobin. 14. 25-33. $10.3109 / 03630269009002252$.
[15] Violeta Yordanova, Todor Tcherkezov - Pilot study of bthalassemia carriers in the region of Kardzali and surroundings - medical and social aspects Molecular-genetics laboratory MBAL "D-r Atanas Dafovski" Kardzali, Bulgaria; EIGHTH INTERNATIONAL MEDICAL CONGRESS OF THE SOUTHEAST EUROPEN MEDICAL FORUM (SEEMF) Dates: 07-10 September 2017 Venue: Athens, Greece.

[16] Felicity K. Boardman and Rachel Hale. I didn't take it too seriously because I'd just never heard of it": Experiential knowledge and genetic screening for thalassaemia in the UK J Genet Couns. 2019 Feb; 28 (1): 141-154. Published online 2018 Dec 24. doi: 10.1002/jgc4.1042.

[17] Cindy-Lee Dennis, Flavia Marini, Jennifer Abbass Dick, Stephanie Atkinson, Jon Barrett, Rhonda Bell, Anick Berard, Howard Berger, Hillary K Brown, Evelyn Constantin, Deborah Da Costa, Andrea Feller, Astrid Guttmann, Magdalena Janus, K S Joseph, Peter Jüni, Sarah Kimmins, Nicole Letourneau, Patricia Li, Stephen Lye, Jonathon L Maguire, Stephen G Matthews, David Millar, Dragana Misita, Kellie Murphy, Anne Monique Nuyt, Deborah L O'Connor, Rulan Savita Parekh, Andrew Paterson, Martine Puts, Joel Ray, Paul Roumeliotis, Stephen Scherer, Daniel Sellen, Sonia Semenic, Prakesh S Shah, Graeme N Smith, Robyn Stremler, Peter Szatmari, Deanna Telnner, Kevin Thorpe, Mark S Tremblay, Simone Vigod, Mark Walker, Catherine Birken BMJ Open. 2021; 11 (2): e46311. Published online 2021 Feb 10. doi: 10.1136/bmjopen-2020-046311 Protocol for a randomised trial evaluating a preconception-early childhood telephone-based intervention with tailored e-health resources for women and their partners to optimise growth and development among children in Canada: a Healthy Life Trajectory Initiative (HeLTI Canada).

[18] Alexandra L. Foulkes, Takahiro Soda, Martilias Farrell, Paola Giusti-Rodríguez, and Gabriel Lázaro-Muñoz LEGAL AND ETHICAL IMPLICATIONS OF CRISPR APPLICATIONS IN PSYCHIATRY* ${ }^{*}$. 